Of the 16 patients advised to self-refer to LCW, 5 did so- a completed referral rate of $31.3 \%$.

Conclusion. The completed referral rate of $31.3 \%$ is difficult to interpret given there are no standards in this area. On one hand, the self-referral process as it currently exists is functioning; on the other, some two-thirds of patients are not making the most of a service deemed to be of benefit to their recovery.

To improve completed referral rates, efforts should be made to better 'sell' LCW to the patient. Potential ways of doing this would be through closer working with LCW- for example, LCW could join the clinical meetings more regularly to discuss new services they offer and feedback any patients A\&L has referred. There should also be emphasis on making the self-referral process as straightforward as possible.

A secondary finding was the notable monthly variation in advised referral rates. It is important to ensure the A\&L team are consistently identifying the right patients for LCW, and again, closer liaison with LCW would help achieve this.

\section{Clozapine \& constipation: an audit of bowel habit monitoring and laxative prescribing in inpatients on clozapine}

Holly Boyd ${ }^{\star}$ and Anna Manso de Zuniga

Merseycare NHS Foundation Trust

${ }^{*}$ Corresponding author.

doi: 10.1192/bjo.2021.220

Aims. To establish how often bowel habits are monitored in inpatients on clozapine

To determine how many of these patients are prescribed laxatives and whether these are utilised

Background. It's estimated that $30-60 \%$ of patients will suffer from constipation whilst on clozapine; this can lead to ileus, intestinal obstruction and bowel ischaemia, all of which can be fatal. Constipation is much more common than clozapine-induced blood dyscrasias, and has a higher mortality rate. Despite this, there is no strict universal framework for bowel habit monitoring equivalent to the compulsory FBC monitoring. Local trust guidance indicates that bowel habits should be monitored regularly, at least at any point of blood sampling. However, monitoring processes across the trust were noted to be variable, as were laxative prescribing practices.

Method. The data sample of current inpatients on clozapine across the trust was identified from pharmacy records. The patient's Rio notes from the preceding 3 months were searched for predetermined terms relating to bowel habits and constipation, and the notes were then analysed for assessment of bowel habit. The number of FBCs collected during this 3 month period was then used to produce comparison with the audit standard. The data on laxative prescribing were collected from current medication lists on EPMA.

Result. A data sample of 31 current inpatients was identified. The audit found that only $54.8 \%$ (17) of patients had their bowel habits monitored at least with every FBC taken. There was significant variability between different wards, with the best performing ward having $100 \%$ adherence to the audit standard, and the worst performing having $0 \%$. In terms of laxative prescribing, it was found that $87.1 \%$ (27) of patients had at least 1 regular or 1 PRN laxative prescribed. Regular laxatives were prescribed for $61.2 \%$ (19) of patients, whereas only PRN laxatives were prescribed in $25.8 \%$ (8) of patients. Of those prescribed only PRN laxatives, only $50 \%$ (4) ever utilised this medication.
Conclusion. Bowel habits are not consistently monitored across the trust in inpatients on clozapine, leaving room for potentially life-threatening side effects to be missed. Additionally, regular laxative prescribing is not standard throughout the trust, which could further add to the potential for significant constipation-related morbidity to occur. A standard method of monitoring bowel habits throughout the trust, as well as a trust laxative prescribing policy, could be a way of remedying this issue and preventing harmful outcomes for our patients on clozapine.

\section{Oppurtunistic completion of the 9 diabetic care processes during inpatient admission to a mental health hospital: an audit of local practice}

Chad Brooker-Thompson*, Yasmin Sultana and Adeela Ashraf Goodmayes Hospital, North East London NHS Foundation Trust ${ }^{\star}$ Corresponding author.

doi: 10.1192/bjo.2021.221

Aims. Diabetes is more prevalent in people with mental illness than in the general population. Those with both mental illness and diabetes are more likely to have poor glycaemic control. Clients with mental illness and diabetes are less likely to receive the 9 NICE recommended annual diabetic care processes than the general population. In 2017, the Joint British Diabetes Societies for Inpatient Care (JBDS-IP) and the Royal College of Psychiatrists released guidance recommending that inpatient psychiatric admissions should be used as an opportunity to complete diabetic care processes, and a named staff member should be responsible for this.

We aimed to review local compliance with this JBDS-IP guidance, increase knowledge and improve local care for clients living with both mental illness and diabetes.

Method. We reviewed the notes of all current inpatients to general, forensic or learning disability wards at our centre and identified all patients with a known diagnosis of Diabetes. We identified which of the 9 care processes had been completed (or had the most recent result documented, or had a plan made for completion) during this admission. We identified if a named staff member was responsible for completing processes on each ward, and whether the care processes were documented in the patients' notes.

Result. We identified 18 current diabetic inpatients at our centre (14\% of inpatients). We found that none of these patients had a diabetic care processes review documented and none of these patients had had a foot check and urinary albumin performed during admission, or had the last community result identified and documented. We found that less than $15 \%$ of patients had a documented plan concerning the completion of retinal screening. One ward had a named staff member responsible for reviewing their diabetic patients' screening. However, 6/9 care processes had been completed in the significant majority of patients $(>75 \%)$.

Conclusion. Our centre is not compliant with the guideline audited. We have implemented a plan to increase awareness of care processes through posters, teaching (at junior and consultant level), creating documentation templates and ensuring wards nominate a staff member to review care processes. We have organised a re-audit. Organising foot examination, renal function testing and retinal screening during admission for clients who may have complicated social situations and may not be aware of (or be non adherent with) the long term management of their diabetes has the potential to significantly reduce morbidity in this client subgroup. 